\title{
Harmonic Analysis on SL(2, R) and Smoothness of the Density of States in the One-Dimensional Anderson Model
}

\author{
Barry Simon $^{1 \star}$ and Michael Taylor ${ }^{2 \star \star}$
}

1 Division of Physics, Mathematics and Astronomy, California Institute of Technology, Pasadena, California 91125, USA

2 Department of Mathematics, State University of New York at Stony Brook, Stony Brook, New York 11794, USA

\begin{abstract}
We consider infinite Jacobi matrices with ones off-diagonal, and independent identically distributed random variables with distribution $F(v) d v$ on-diagonal. If $F$ has compact support and lies in some Sobolev space $L_{\alpha}^{1}$, then we prove that the integrated density of states, $k(E)$, is $C^{\infty}$ in $E$.
\end{abstract}

\section{Introduction}

In this paper, we will study the one-dimensional Anderson model

$$
\left(h_{\omega} u\right)(n)=u(n+1)+u(n-1)+V_{\omega}(n) u(n)
$$

on $l^{2}(Z)$, where $V_{\omega}(n)$ are independent identically distributed random variables with distribution $d \eta(v)$. The operator restricted to $l^{2}([0, l-1])$ with $u(-1)=u(l)=0$ boundary condition is denoted by $h_{\omega}^{l}$. This $l \times l$ matrix has eigenvalues $e_{\omega}^{l}(1)<\cdots$ $<e_{\omega}^{l}(l)$. The integrated density of states, $k(E)$, is defined by

$$
k(E)=\lim _{l \rightarrow \infty} l^{-1} \#\left(j \mid e_{\omega}^{l}(j)<E\right) .
$$

It is a basic result $[3,2,11]$, essentially a consequence of the ergodic theorem, that for a.e. $\omega$ the limit exists for all $E$.

It is a result of Pastur [15] that $k(E)$ continuous in $E$, Craig-Simon [6] show that $k$ is Log-Hölder continuous, i.e. $\left|k(E)-k\left(E^{\prime}\right)\right| \leqq c_{R}\left\{\ln \left(\left|E-E^{\prime}\right|\right\}^{-1}\right.$ if $|E| \leqq R,\left|E-E^{\prime}\right|<\frac{1}{2}$, and LePage [12] that $k(E)$ is Hölder continuous of some order $\alpha>0$ in this situation. (The results of $[6,15]$ hold in great generality.) Here we want to consider greater regularity in $E$. Without restrictions on $d \eta$, one cannot expect too much more regularity. There is an argument of Halperin [24], essentially

* Research partially supported by USNSF under Grant MCS-81-20833

$\star \star$ Research partially supported by USNSF under Grant MCS-82-01766A01 
already rigorous, that when $d \eta(v)=\theta \delta(v-a)+(1-\theta) \delta(v-b)$ for $a, b, \theta$ suitable, then $k$ is not $C^{1}$; indeed, for any $\alpha>0$, there are $a, b, \theta$ so that $k$ is not Hölder continuous of order $\alpha$. Since this argument is not widely known in the mathematical community, since Halperin's model is slightly different from this discrete model, and since he does not deal with some unessential points of rigor, we provide a formal version of his result in Appendix 3. Thus, we will restrict ourselves to the case where $d \eta$ is absolutely continuous,

$$
d \eta(v)=F(v) d v .
$$

We are especially interested in the case originally discussed by Anderson, where $F$ is a multiple of the characteristic function of an interval.

We will need a weak regularity condition on $F$, expressed in terms of Sobolev spaces defined by: $L_{\alpha}^{p}\left(R^{v}\right) 1 \leqq p \leqq \infty ; \alpha \geqq 0$ is the set of $f \in L^{p}$ so that there is a $g \in L^{p}$ with the Fourier transforms related by $\hat{g}(k)=\left(1+k^{2}\right)^{\alpha / 2} \hat{f}(k)$. The properties that we need of these spaces are discussed in Appendix 1. We remark now that if $f$ has compact support and $f \in L_{\alpha}^{p}$, then $f \in L_{\alpha}^{1}$, and that if $f$ is the characteristic function of an interval, then $f \in L_{\alpha}^{2}$ for $0 \leqq \alpha<\frac{1}{2}$, and so in $L_{\alpha}^{1}$ for $0 \leqq \alpha<\frac{1}{2}$ (actually, one can take $\alpha<1$ for $L_{\alpha}^{1}$ ). Our main result in this paper is:

Theorem 1.1. If $F$ has compact support and $F \in L_{\alpha}^{1}$ for some $\alpha>0$, then $k(E)$ is a $C^{\infty}$ function of $E$.

In particular, this result applies if $F$ is a multiple of the characteristic function of an interval. There is some previous evidence that $k(E)$ is $C^{\infty}$ in this case. If $F(x)=$ $(b-a)^{-1} \chi_{(a, b)}(x)$, then the points of increase of $k(E)$ are precisely on $[a-2, b+2]$ but near $a-2$ (and similarly, near $b+2), k(E)$ goes to zero as $\exp \left(-c(E-a+2)^{-1 / 2}\right)$ $[7,13,14,17,19]$. These "Lifschitz tails" are suggestive that $k$ is $C^{\infty}$ : At least at the points where all derivatives from the left vanish, the behavior on the right is consistent with the smoothness of $k$. One can also see evidence of the smoothing nature of putting $V$ into a Jacobi matrix by directly computing the average over $V_{\omega}$ of the \#(j|e $\left.e_{\omega}^{l=2}(j)<E\right)$. One finds this density $k^{(2)}(e)$ has the form $\int_{-\infty}^{e} F^{(2)}(v) d v$, where $F^{(2)}$ is now continuous (unlike $F^{(1)}(v)=(b-a)^{-1} \chi_{(a, b)}(v)$ ), but $d F^{(2)} / d v$ is discontinuous at the points $a \pm 1, b+1, \frac{1}{2}(b+a) \pm \sqrt{\left(\frac{a-b}{2}\right)^{2}+1}$. In fact, our proof of Theorem 1.1 shows that $k^{(l)}(e)=\int_{-\infty}^{e} F^{(l)}(v) d v$, with $F^{(l)}(v)$ having more and more derivatives as $l$ gets larger and larger.

In Sect. 2, following Schmidt [18], we show that smoothness of $k$ in $E$ is connected to smoothness in $E$ of the invariant measure on $\mathrm{PR}(1)$ (the projective line) associated to the "transfer matrix" for $h_{\omega} u=E u$. We will discuss the reason why the attempt to analyze this measure directly appears to fail, and forces us to convolutions on $\operatorname{SL}(2, R)$. In Sect. 3, we analyze this problem by using convolutions on $\operatorname{SL}(2, R)$, and reduce the proof of Theorem 1.1 to a result on the three-fold convolution of the measure on $\operatorname{SL}(2, R)$ given by $\left[\begin{array}{cr}e-v & -1 \\ 1 & 0\end{array}\right]$, where $v$ has distribution $F(v) d v$. In Sect. 4, we prove this technical result, thereby completing the 
proof of Theorem 1.1. We provide appendices on $L_{\alpha}^{p}$ and $\operatorname{SL}(2, R)$ for the reader's convenience.

While we will discuss the smoothness of the density of states appropriate to the electronic Hamiltonian, the same arguments prove smoothness of the density appropriate to a random harmonic chain; that is, we seek solutions of

$$
u_{n+1}+u_{n-1}+\left(m_{n} \omega^{2}-2\right) u_{n}=0,
$$

where $m_{n}$ is now the random variable and the "spectrum" is the set of values of $\omega$. Again (up to signs) the integrated density of states is given by the weight an invariant measure on $\{\theta \in(0,2 \pi])$ gives to $(0, \pi)$, so smoothness of the invariant measure yields smoothness of $\widetilde{k}\left(\omega^{2}\right)$. Essentially one can consider the invariant measures on RP(1) associated to the measure $d \mu_{\lambda, E}$ on $\operatorname{SL}(2, R)$ given by $\left[\begin{array}{cc}E-\lambda V & -1 \\ 1 & 0\end{array}\right]$, where $V$ has distribution $d \kappa(v)$. The electronic case corresponds to smoothness in $E$ for $\lambda=1$, while the harmonic chain corresponds to smoothness in $\lambda$ for $E=2$. It is easy to show that the same hypotheses on $v$ that yield smoothness of $k(E)$ also yield smoothness of $\tilde{k}$ if $m$ has the distribution $d \kappa$.

\section{Invariant Measures on the Real Projective Line}

The key to relating the behavior of $k(e)$ to invariant measures is the following version of the Sturm oscillation theorem:

Theorem 2.1. Fix a potential $V$, and let $u$ be any non-zero solution of

$$
u(n+1)+u(n-1)+(V(n)-E) u(n)=0 .
$$

Let $i_{u}(n)=1$ if $u(n) u(n+1)>0$, or if $u(n)=0$ and $i_{u}(n)=0$ otherwise. Let $e_{v}^{l}(j)$ be the eigenvalues of $h^{l}$. Then

$$
\lim _{l \rightarrow \infty} l^{-1}\left|\#\left\{j \mid e_{v}^{l}(j)<E\right\}-\sum_{n=0}^{l-1} i_{n}(n)\right|=0 .
$$

Proof. Let $\tilde{u}$ be a solution of (2.1) obeying $u(-1)=0$. Then the discrete Sturm oscillation theorem [1] says that

$$
\#\left(j \mid e_{v}^{l}(j)<E\right)=\sum_{n=0}^{l-1} i_{\tilde{u}}(n)
$$

A comparison theorem implies that

$$
\left|\sum_{n=0}^{l-1} i_{u}(n)-i_{\hat{u}}(n)\right| \leqq 1
$$

which implies (2.2).

Remark. The reader familiar with the continuum case may be surprised that we count non-sign flips $(u(n) u(n+1)>0)$ rather than sign-flips $(u(n) u(n+1)<0$; equivalently the linear interpolation of $u$ to $R$ has a zero in $(n, n+1))$. This is because the discrete analog of $-d^{2} / d x^{2}$ has a $-(u(n+1)+u(n-1))$ term, so that the direct discrete analog of Sturm oscillation counts sign flips to get \# $\left(j \mid e_{v}^{l}(j)>E\right)$. 
Define $x(n)=u(n+1) / u(n) \in \dot{\mathbb{R}} \equiv$ one point compactification of $\mathbb{R}$, so $x$ obeys

$$
x(n)=E-V(n)-x(n-1)^{-1},
$$

and $i_{u}(n)=\chi_{(0, \infty]}(x(n))$ with $\chi_{(0, \infty)}$ the characteristic function of $(0, \infty]$ in $\dot{\mathbb{R}}$.

Consider now a random potential $V(n)$. For each choice $x_{0}$ of boundary condition and each sample potential $V_{\omega}(n)$, let $x_{n}\left(\omega, x_{0}\right)$ solve $(2.3)$ with $V(n)=V_{\omega}(n)$ and $x(0)=x_{0}$. Since $l^{-1} \#\left(j \mid e_{\omega}^{l}(j)<E\right)$ is bounded and the limit is $k(E)$ for a.e. $\omega$, we see that:

Corollary 2.2. For each fixed $x_{0}$ :

$$
k(E)=\lim _{l \rightarrow \infty} l^{-1} \sum_{n=0}^{l-1} \operatorname{Exp}\left(\chi_{(0, \infty)}\left(x_{n}\left(\omega, x_{0}\right)\right)\right) .
$$

Since this holds for each $x_{0}$ and the quantity on the right of (2.4) is bounded, (2.4) holds if $x_{0}$ is also a random variable, and if Exp then takes the meaning of expectation independently over $V_{\omega}$ and $x_{0}$. A distribution $d v$ for $x_{0}$ is called invariant if $x_{1}\left(\omega, x_{0}\right)$ has distribution $d v$ also. Since $V_{\omega}$ is i.i.d., it is easy to see then that $x_{n}\left(\omega, x_{0}\right)$ also has distribution $d v$, so (2.4) implies

Theorem 2.3. (Schmidt [18]). If $d v_{E}$ is an invariant measure for (2.3), then

$$
k(E)=\int \chi_{(0, \infty]}(x) d v_{E}(x) .
$$

This result says that smoothness in $E$ will follow from suitable smoothness of $v_{E}$ in $E$. If $V$ has distribution $F(v) d v$ and $x_{0}$ has distribution $d v$, then $x_{1}\left(\omega, x_{0}\right)$ has distribution $d \mu(y)=G(y) d y$, where

$$
G(y)=\int F\left(e-y-x_{0}^{-1}\right) d v\left(x_{0}\right)=\tilde{T}(v)(y) .
$$

We remark that (2.6) is not quite intended literally: If $d v$ has no pure point piece at $x_{0}=0$, then $d \mu(y)$ is $G(y) d y$ with $G$ given by (2.6). If $d v=\alpha \delta_{x_{0}=0}+d \tilde{v}$, where $d \tilde{v}$ has no pure point at 0 , then $d \mu=\alpha \delta_{y=\infty}+\widetilde{T}(\tilde{v}) d y$ with $\widetilde{T}$ given by (2.6). Thus, applying $\widetilde{T}$ twice, we also get a measure of the form $G(y) d y$, so to look for fixed points of $v \mapsto u$, we need only look for fixed points of the map

$$
\left(T_{e} G\right)(y)=\int F\left(e-y-x_{0}^{-1}\right) G\left(x_{0}\right) d x_{0} .
$$

The naive strategy is now clear: Let $G_{e}$ be the solution of $T_{e} G_{e}=G_{e}$. We want to show that $G_{e}$ is smooth in $e$ (there is a problem associated with the non-compactness of $[0, \infty)$ if one doesn't specify in which space $G_{e}$ is proven smooth; we will eventualy study the transform of $T_{e}$ acting on a compact space). As we shall see, it is a theorem of Furstenberg that 1 is a simple eigenvalue of $T_{e}$. Thus the following result is of some interest:

Proposition 2.4. Let $d \mu_{0}$ be a probability measure on a space $X$. Let $\left\{A_{e}\right\}_{e \in I}$ be a family of compact operators on $L^{2}(X, d \mu)$ for $e$ in an open interval $I$, so that;

(1) 1 is an (algebraically) simple eigenvalue of $A_{e}$ for each $e \in I$.

(2) The eigenvector $\varphi_{e}$ associated to $A_{e}$ with eigenvalue 1 has $\int \varphi_{e}(x) d \mu(x) \neq 0$, so we can normalize $\varphi_{e}$ by

$$
\int \varphi_{e}(x) d \mu(x)=1
$$


(3) $A_{e}$ is $C^{l}$ as operators on $L^{2}$.

Then $\varphi_{e}$ is $C^{l}$ as vectors on $L^{2}$.

Proof. Let $[a, b] \subset I$. By compactness of $A_{e}, 1$ is an isolated eigenvalue of $A_{e}$, so there exists $\varepsilon$ so that if $e \in[a, b]$ and $0<|\lambda-1|<2 \varepsilon$, then $\lambda \notin \operatorname{spec}\left(A_{e}\right)$. Fix $e_{0} \in[a, b]$ and let $\psi=\varphi_{e_{0}}$. Then for $e$ near $e_{0}, \varphi_{e}$ can be written:

$$
\begin{aligned}
& \varphi_{e}=P_{e} \psi /\left(1, P_{e} \psi\right), \\
& P_{e}=(2 \pi i)^{-1} \oint_{|\lambda-1|=\varepsilon}\left(\lambda-A_{e}\right)^{-1} d \lambda .
\end{aligned}
$$

Since $A_{e}$ is $C^{l},\left(\lambda-A_{e}\right)^{-1}$ is $C^{l}$ and thus $P_{e}$ is $C^{l}$. It follows that $\varphi_{e}$ is $C^{l}$.

The above result is not directly applicable to $T_{e}$. The fact that we don't have a probability measure will be treated by shifting the background measure from $d x$ to $\pi^{-1}\left(1+x^{2}\right)^{-1} d x . T_{e}$ is not compact (it can't be, since it maps $\delta_{x=0}$ to $\delta_{x=\infty}$ ), but $T_{e}^{2}$ is compact. The key point is that if $F$ isn't infinitely smooth, then $T_{e}$ cannot be infinitely smooth. But $T_{e}^{l}$ has an integral kernel given by a kind of twisted convolution, so one can hope that as $l$ increases, $T_{e}^{l}$ become smoother and smoother in $e$. For example, if $F$ is only assumed in $C^{1}$, then one might hope to prove that the kernel of $T_{e}^{2}$,

$$
T_{e}^{2}(y, z)=\int F\left(e-y-x^{-1}\right) F\left(e-x-z^{-1}\right) d x
$$

is $C^{2}$ by formally taking second derivatives, but then shifting second derivatives on one $F$ to first derivatives on each $F$ by a change of variables and integration by parts. This procedure does not work: The $x^{-1}$ factors resulting from the change of variables make various integrals absolutely divergent. In a sense we will eventually make precise, one wants to integrate by parts not a whole time, but at most a third of a time. This is hard to do in the setting of functions on $R$ with a "sort of convolution," but will be easy if we shift to $\operatorname{SL}(2, R)$ where "sort of convolution" becomes legitimate convolution. The fact that the naive approach fails should motivate the more abstract framework of the next section.

Before ending this section, we want to realize $\dot{\mathbb{R}}$ in an equivalent way as $R P(1)$, the real projective line. That is, we set $x=\tan \theta ; \theta \in[-(\pi / 2), \pi / 2]$ with $\pi / 2$ and $-(\pi / 2)$ associated to a point. This is equivalent to considering the line $l_{\theta}=$ $\left\{r\left(\begin{array}{c}\sin \theta \\ \cos \theta\end{array}\right) \mid r \in R \backslash\{0\}\right\} \in R P(1)$, the set of all $l_{\theta}$. Any $2 \times 2$ real matrix, $A$, induces a map, $\tilde{A}$, on $R P(1)$ by $A l_{\theta}=l_{\tilde{A} \theta}$.

Given $F$, define the measure $d \mu^{(F, E)}$ on $\operatorname{SL}(2, R)$ to be the measure on matrices of the form $\left[\begin{array}{cr}E-v & -1 \\ 1 & 0\end{array}\right]$ where $v$ has distribution $F(v) d v$. Theorem 2.3 then becomes:

Theorem 2.3'. Suppose that $d v_{E}$ is a measure on $R P(1)$ so that if $A$ and $\theta$ are independently distributed with $\theta$ having distribution $d v_{E}$ and $A$ having distribution $d \mu^{(F, E)}$, then $\tilde{A} \theta$ has distribution $d v_{E}$. Then $k(E)=\int \chi_{(0, \pi / 2]}(\theta) d v_{E}(\theta)$.

Corollary 2.5. If $d v_{E}$ has the form $H(\theta, E) d \theta$ with $H C^{\infty}$ in $\theta$ and $E$, then $k(E)$ is $C^{\infty}$.

We note that the Thouless formula $[10,23,2,6]$ relates the Lyaponov exponent, $\gamma(E)$, to $k(E)$ by

$$
\gamma(E)=\int \ln \left|E-E^{\prime}\right| d k\left(E^{\prime}\right)
$$


so that smoothness of $k$ in $E$ implies smoothness of $\gamma$ in $E$. Actually, one can obtain this also from smoothness of $H(\theta, E)$ in $E$ without recourse to the Thouless formula since one has [9]:

$$
\gamma(E)=\frac{1}{2} \int F(v) H(\theta, E) \ln \left\{[(E-v) \sin \theta-\cos \theta]^{2}+\sin ^{2} \theta\right\} d \theta d v .
$$

\section{Formulation on $\operatorname{SL}(2, R)$ : Reduction to the Main Technical Result}

Following Furstenberg $[8,9]$, one can formulate the condition defining $v_{E}$ most naturally in terms of convolutions on $\operatorname{SL}(2, R)$ and on $R P(1)$. Given a measure $\mu$ on $\mathrm{SL}(2, R)$ and a measure $\gamma$ on $P R(1)$, we define a measure $\mu * \gamma$ on $P R(1)$ by

$$
\int f(\theta) d(\mu * \gamma(\theta))=\int f(\widetilde{A} \theta) d \mu(A) d \gamma(\theta) .
$$

Thus, if $d \mu^{(F, E)}$ is the measure on $\operatorname{SL}(2, R)$ described at the end of the last section, and if $T_{E}: \mathscr{M}(R P(1)) \rightarrow \mathscr{M}(R P(1))(\mathscr{M}(\cdot) \equiv$ measures on $\cdot)$ is defined by

$$
T_{E}(\gamma)=\mu^{(F, E)} * \gamma
$$

then $\gamma_{E}$ is determined by

$$
T_{E}\left(v_{E}\right)=v_{E} .
$$

In this regard, the following theorem of Furstenberg $[8,9]$ is important.

Theorem 3.1. Let $\mu$ be an arbitrary probability measure on $\operatorname{SL}(2, R)$ with the property that there is no measure $\gamma$ on $R P(1)$ obeying $\delta_{A} * \gamma=\gamma$ for each A Esupp $\mu$. Then there is a unique probability measure, $v$, on $R P(1)$ obeying $\mu * \gamma=\gamma$.

Remarks. 1. $\delta_{A}$ is the point measure on $\operatorname{SL}(2, R)$ concentrated at $A$.

2. The result in $[8,9]$ involves $\operatorname{SL}(n, R)$ and $R P(n-1)$.

3. If [ $\operatorname{supp} \mu]$ is the smallest closed subgroup containing supp $\mu$, clearly $\delta_{A} * \gamma=\gamma$ for all $A \in[\operatorname{supp} \mu]$.

4. In particular, if $[\operatorname{supp} \mu]=\operatorname{SL}(2, l), \mu$ has the required property since $R P(1)$ supports no measure invariant under each $\delta_{A}$.

We need the following consequence of Furstenberg's theorem:

Corollary 3.2. If $\mu$ has the above property, if $T(\gamma)=\mu * \gamma$ and 1 is an isolated point of $\operatorname{spec}(T)$, then 1 has algebraic multiplicity 1.

Proof. The theorem says that $T$ has geometric multiplicity one. We must show that there is no $\gamma \in M(R P(1))$ obeying

$$
T \gamma=\gamma+v
$$

There is no such $\gamma$, since $\int T(\gamma)=\int \gamma$, so (3.3) would imply $\int v=0$, which is not true.

Following the idea in the last section, we want to take powers of $T_{E}$. In this context, one wants to define convolution on $\operatorname{SL}(2, R)$. If $\mu, \kappa$ are two measures on $\operatorname{SL}(2, R)$, we define the measure $\mu * \kappa$ on $\operatorname{SL}(2, R)$ by

$$
\int f(A) d(\mu * \kappa)(A)=\int f(A B) d \mu(A) d \kappa(B) .
$$


The fact that $(A B)^{\tilde{A}}=\widetilde{A} \widetilde{B}$ implies that if $\mu, \kappa \in M(\operatorname{SL}(2, R)), \gamma \in M(R P(1))$,

$$
(\mu * \kappa) * \gamma=\mu *(\kappa * \gamma) \text {. }
$$

In (3.4), one * is an $\operatorname{SL}(2, R)$ convolution and the others are $\operatorname{SL}(2, R) \times R P(1)$ convolutions. This associative law justifies the use of one symbol for both convolutions.

Thus, powers of $T_{E}$ are just convolution powers of $\mu^{(F, E)}$. We let $* l \mu$ denote the $l$ fold convolution of $\mu$. In this section, we want to first reduce the proof of our main result, Theorem 1.1 , to a result on ${ }^{* l} \mu$, and then reduce the proof of this result to a technical fact proven in the next section. The result on $* l \mu$ is:

Theorem 3.3. Let $F \in L_{\alpha}^{1}(R)(\alpha>0)$ with compact support. For any $k$, there exists $l_{0}$ (depending only on $\alpha$ and $k$ ) so that, for $l \geqq l_{0},{ }^{* l} \mu^{(F, E)}$ is absolutely continuous relative to Haar measure, $d A$, on $\operatorname{SL}(2, R)$ and

$$
d\left({ }^{* l} \mu^{F, E}\right)=G_{l}(A, E) d A,
$$

where $G_{l}$ has compact support in $A$ and is $C^{k}$ jointly in $E$ and $A$.

As explained above, we will first show how Theorem 3.3 implies Theorem 1.1. We need the following:

Lemma 3.4. If $\kappa$ is a measure on $\operatorname{SL}(2, R)$ of the form $G(A) d A$, with $G$ a $C^{k}$ function of compact support, then $T(\kappa)$, the map on $L^{2}(R P(1), d \theta)$ defined by $\kappa *(f d \theta)=(T(\kappa) f) d \theta$ obeys

(a) $T$ is Hilbert Schmidt.

(b) Any eigenfunction of $T$ is $C^{k}$ in $\theta$; in fact, $T$ is a bounded map from $L^{2}$ into $C^{k}$ functions (in the natural $C^{k}$ topology).

(c) Any solution of $\kappa * \gamma=x \gamma$, some complex $x$ obeys $\gamma=G d \theta$, with $G$ a $C^{k}$ function.

Proof. Let $X$ be the map from $\operatorname{SL}(2, R) \times R P(1)$ to $R P(1)$ by $X(A, \theta)=\tilde{A} \theta$. A direct calculation shows that $X$ is $C^{\infty}$, and that at $A=i d$ (where $d a_{11}=-d a_{22}$ ) we have

$$
\left.d X(A, \theta)\right|_{d \theta=0} ^{A=i d}=(\sin 2 \theta) d a_{11}+\cos ^{2} \theta d a_{12}-\sin ^{2} \theta a_{21}
$$

so $\left.\nabla_{A} X\right|_{A=i d} \neq 0$. Since $X(A, \theta)=X\left(A A_{0}^{-1}, A_{0} \theta\right)$, we see that globally $\nabla_{A} X \neq 0$. The formula for the integral kernel of $T(\kappa)$ :

$$
T(\kappa)\left(\theta, \theta^{\prime}\right)=\int g(A) \delta\left(X\left(A, \theta^{\prime}\right)-\theta\right) d A
$$

shows that $T$ has a bounded integral kernel (so (a) holds), and $T\left(\theta, \theta^{\prime}\right)$ is $C^{k}$ in $\theta$ uniformly in $\theta^{\prime}$, so $T$ is actually continuous from measures on $R P(1)$ to $C^{k}$, proving (b) and (c).

Proof of Theorem 1.1 (assuming Theorem 3.3). By Corollary 2.5, we need only show that the solution of $T\left(\mu^{(F, E)}\right) v_{E}=v_{e}$ has form $H(\theta, E) d \theta$ with $H C^{\infty}$ in $\theta$ and $E$. Set $T_{E}=T\left(\mu^{(F, E)}\right)$. By the last lemma, $T_{E}^{l}$ is a family of compact operators on $L^{2}$. Corollary 3.2 is applicable since $\left[\operatorname{supp}^{* l} \mu\right]$ is all of $\operatorname{SL}(2, R)$. By Corollary $3.2,1$ has algebraic multiplicity 1 (for each $l$ ), and by the last lemma and Theorem $3.3, T_{E}^{l}$ is $C^{k}$ in $E$. Thus, Proposition 2.4 applies and $v_{E}$ is $C^{k}$ in $E$ (as elements in $L^{2}$ ). Since $T_{E}^{l}$ maps $L^{2}$ to $C^{k}$ (in $\theta$ ), and $d^{r} T_{E}^{l} / d E^{r}(r=0,1, \ldots, k)$ maps $L^{2}$ to $C^{k}$ (by Theorem 3.3 and 
the last lemma), $T_{E}^{l} v_{E}$ is jointly $C^{k}$ in $\theta$ and $E$, i.e. $H$ is $C^{k}$. Since $k$ is arbitrary, $H$ is $C^{\infty}$.

Our proof of Theorem 3.3 will exploit the use of Sobolev spaces on $\operatorname{SL}(2, R)$. An alternate proof might be possible by using the Fourier transform on $\operatorname{SL}(2, R)$, but because of the complicated calculations required of such a procedure, we have not used that approach. If such a proof worked, it might well extend the theorem to one where the hypothesis could be replaced by a requirement $|\hat{F}(k)| \leqq C\left(1+k^{2}\right)^{-\delta}$ (for some $\delta>0$ ) which would include some continuous singular $d \eta(v)$. Such a procedure also might allow one to drop the hypothesis that $F$ has compact support.

There is a technical complication on $\operatorname{SL}(2, R)$ that the right and left invariant Laplacians are unequal, and differ in a significant way at infinity (see Appendix 2); we finesse this difficulty by dealing everywhere with objects of compact support. The translations are defined on functions on $\operatorname{SL}(2, R)$ by $\left(\tau_{A}^{L} f\right)(B)=f\left(A^{-1} B\right),\left(\tau_{A}^{R} f\right)(B)$ $=f(B A)$. There exist "Laplacians" $\Delta_{L}, \Delta_{R}$ (depending on a choice of metric on the Lie algebra $\operatorname{SL}(2, R)$ with $\Delta_{L}$ commuting with all $\tau_{A}^{L}$, etc. These operators are essentially selfadjoint on $C_{0}^{\infty}(\operatorname{SL}(2, R))$, so $(1-\Delta)^{s / 2}$ is defined by the spectral theorem. The Sobolev spaces, $H^{s}$, are defined for $s \geqq 0$ by $g \in H^{s}$ if and only if $g \in L^{2}$ and $\left(1-\Delta_{L}\right)^{s / 2} g \in L^{2}$ with $\|g\|_{s}=\left\|\left(1-\Delta_{L}\right)^{s / 2} g\right\|_{L^{2}}$. For $s<0, H^{s}$ is defined by duality in the usual way. For $K$ compact, let $H_{K}^{s} \equiv\left\{f \in H^{s} \mid \operatorname{supp} f \subset K\right\}$, and define $H_{\text {comp }}^{s}$ $=\bigcup_{K \text { compact }} H_{K}^{s}$. To say a map $S: \mathscr{D}^{\prime} \rightarrow \mathscr{D}^{\prime}$ maps $H_{\text {comp }}^{s}$ to $H_{\text {comp }}^{r}$ means that for each $K$, there exists $\tilde{K}$ compact and a constant $C(K)$ so that $S$ maps $H_{K}^{s}$ to $H_{\widetilde{K}}^{r}$ and for $f \in H_{K^{\prime}}^{s}$

$$
\|S f\|_{r} \leqq c(K)\|f\|_{s} .
$$

It is not hard to see that if $f$ has support in $K$, then $f \in H_{K}^{s}$ if and only if $f$ is an $H^{s}$ function in the usual $R^{3}$ sense in a local coordinate system about each point. In particular, every distribution of compact support lies in some $H^{s}(-\infty<s<\infty)$. By an ordinary Sobolev estimate, for any measure of compact support, $\mu \in H^{s}$, all $s<-3 / 2$ and

Lemma 3.5. For any $F$ of compact support, $d^{r} \mu^{(F, E)} / d E^{r}$ lies in $H^{s}$ for all $s<-\frac{3}{2}-r$.

Proof. We can cover SL(2,R) with two coordinate patches; if $A=\left[\begin{array}{ll}a & b \\ c & d\end{array}\right]$, we take one patch where $b \neq 0$ and use coordinates $a, b, d$ with Haar measure $b^{-1} d a d b d d$, and one patch where $a \neq 0$ with coordinates $a, b, c$ and Haar measure $a^{-1} d a d b d c$. By changing variables in $\int g(A) d \mu^{(F, E)}(A)$ and shifting $E$ into the $a$-coordinate, we see that

$$
\left|\frac{d^{r}}{d E^{r}} \int g(A) d \mu^{(F, E)}(A)\right| \leqq c \sum_{|\alpha| \leqq r}\left\|D^{\alpha} g\right\|_{\infty} \pm C_{s}\|g\|_{-s}
$$

so long as $s<-r-\frac{3}{2}$ by an ordinary Sobolev estimate.

Now define $L_{\alpha}^{p}(\operatorname{SL}(2, R)), p>1$, to be those distributions, $T$, on $\operatorname{SL}(2, R)$ with $|T(f)| \leqq C\left\|\left(1-\Delta_{L}\right)^{-\alpha / 2} f\right\|_{q}$ for some $C$ and all $f \in C_{0}^{\infty}$ and $q$ the dual index of $p$. As above, if $T$ has compact support and $\alpha \geqq 0, T \in L_{\alpha}^{p}$ if and only if $T=t(A) d A$ with $t$ 
locally in the conventional $L_{\alpha}^{p}$ in local coordinates. The following result is proven in Appendix 2.

Theorem 3.6. (a) Let $T \in L_{s}^{p}, 1<p, 0 \leqq s \leqq 1$ with compact support. Then left convolution with $T$ defines a bounded map from $H_{\text {comp }}^{t}$ to $H_{\text {comp }}^{t+s}$ for all $t$.

(b) Let $T \in H^{s}$ have compact support where $s<0$ is allowed. The left convolution with $T$ defines a bounded map of $H_{\mathrm{comp}}^{t}$ to $H_{\mathrm{comp}}^{t+s}$ for all $t$.

The last element we need for the proof of Theorem 3.3 is the following result we prove in the next section:

Theorem 3.7. For each $\alpha>0$, there exists $\alpha^{\prime}>0$ so that the measure $\mu^{(F, E)}$ obeys $\mu^{(F, E)} * \mu^{(F, E)} * \mu^{(F, E)} \in L_{\alpha}^{p},(\mathrm{SL}(2, R))$ if $F \in L_{\alpha}^{1}(R)$ and $p$ is sufficiently close to 1 .

Proof of Theorem 3.3 (Assuming Theorem 3.7). We first show that for sufficiently large $l,{ }^{* l} \mu^{(F, E)}$ has a $C^{k}$ density. By Lemma 3.5, $\mu \in H^{s}$ if $s<-3 / 2$. Thus by Theorems 3.6 and $3.7 *(3 m+1) \mu$ lies in $H^{s}$ if $s<m \alpha^{\prime}-3 / 2$. In particular, if $m$ is sufficiently large, ${ }^{* l} \mu \in H^{s_{0}}$, with $s_{0}>k+3 / 2$ for all $l>3 m+1$. But functions in such an $H^{s_{0}}$ are $C^{k}$ by a Sobolev estimate. Now compute the weak derivative $(d / d E)\left({ }^{* l} \mu^{(F, E)}\right)$ $=\sum_{j=0}^{l-1}(* j \mu) *(d \mu / d E) *\left(*^{l-1-j} \mu\right)$. By Lemma 3.5 and Theorem 3.6, convoluting with $d \mu / d E$ decreases the Hölder index by at most $5 / 2$. If $j=2$, we may not get any extra smoothing from those two factors, so if $l \geqq 3 m+6$, then $(d / d E)\left({ }^{* l} \mu^{(F, E)}\right)$ lies in $H^{t}$ if $t<m \alpha-\psi$. (Note: $\left(4=\frac{3}{2}+\frac{5}{2}\right)$ ) ) Thus, for $l$ sufficiently large, $(d / d E)\left({ }^{* l} \mu\right) \in H^{s_{0}}$ with $s_{0}>k+3 / 2$. By integrating this formal derivative, one sees that the kernel $G_{l}(A, E)$ is Lipschitz in $E$ (uniformly in $A$ ) and that all derivatives $D_{A}^{j} C_{l}(A, E) ; j \leqq k$ are Lipschitz in $E$. By doing the same thing with $\left(d^{*} / d E^{\alpha}\right)\left({ }^{* l} \mu\right) ; \alpha=2, \ldots$, $k+1$, we see that for $l$ large $D_{A}^{j} G_{l}(A, E) ; j \leqq k$ is $C^{k}$; in fact, since ${ }^{* l} \mu$ is $C^{k}$ in $H^{s_{0}}$, one gets that $G_{1}$ is jointly $C^{k}$.

\section{Proof of the Main Technical Theorem}

Our goal in this section is to prove Theorem 3.7. As we shall see, even if $F$ is $C^{\infty}$, the density in $\mu * \mu * \mu$ is not only not $C^{\infty}$ : It is not $C^{1}$ or even in $L_{1}^{p}$ any $p>1$; rather, it will lie in $L_{\alpha}^{p}$ for all $\alpha<1$ and $p>1$ approaching 1 as $\alpha$ approaches 1 . The fact that the derivatives are fractional means that we cannot check $\mu * \mu * \mu \in L_{\alpha}^{p}$ by taking classical derivatives, and indeed we will use complex interpolation (but in an especially simple way). Presumably, if $F$ is $C^{1}$, then $\mu * \mu * \mu * \mu$ lies in $L_{1}^{p}$ (and perhaps it even has $C^{1}$ density), and this could be checked by direct calculation. We deal with the triple convolution here for three reasons: (i) We need to deal with fractional $\alpha$ if we want to have minimal regularity properties on $F$, (ii) The triple convolution is the first which can possible have a density ( $\equiv$ be a.c. with respect to Haar measure), (iii) $\operatorname{SL}(2, R)$ is three dimensional, so the map $\Phi$ below is away from singularities a diffeomorphism; if we took the four-fold convolution, the map would be from $\mathbb{R}^{4}$ to $\mathbb{R}^{3}$ and at best a submersion. This would make explicit calculations more complicated, although we are sure they could be done. 
Note that

$$
\left[\begin{array}{cc}
x & -1 \\
1 & 0
\end{array}\right]\left[\begin{array}{cc}
y & -1 \\
1 & 0
\end{array}\right]\left[\begin{array}{cc}
z & -1 \\
1 & 0
\end{array}\right]=\left[\begin{array}{ll}
a & b \\
c & d
\end{array}\right],
$$

$a=x y z-x-z, b=-x y+1, c=y z-1, d=-y$. Thus, the map $\Phi: \mathbb{R}^{3} \rightarrow \operatorname{SL}(2, R)$ by $\Phi(x, y, z)=\left[\begin{array}{ll}a & b \\ c & d\end{array}\right]$ is basic and $\mu * \mu * \mu$ is just the push forward of the measure $\gamma=G d x d y d z$ with $G(x, y, z)=F(x) F(y) F(z)$ (i.e. $\mu * \mu * \mu(s) \equiv \gamma\left(\Phi^{-1}(s)\right)$ ). Since $F \in$ $L_{\alpha}^{1}(\mathbb{R})$ with compact support, $F \in L_{\alpha-\delta}^{p}$ for some $p>1$ and $\delta$ small (see Theorem A.1.2) so $G \in L_{\alpha-\delta}^{p}\left(\mathbb{R}^{3}\right)$ (see Theorem A.1.7), so we will consider the linear map $\Phi_{*}: G \mapsto \gamma^{\circ} \Phi^{-1}$ and prove that, for $p>1, \alpha>0$, this maps $L_{\alpha, \text { comp }}^{r}$ into some $L_{\alpha^{\prime}}^{p}$ for some $r>1, \alpha^{\prime}>0$.

Proposition 4.1. $\Phi$ is a diffeomorphism in a neighborhood of any point $\left(x_{0}, y_{0}, z_{0}\right)$ with $y_{0} \neq 0$.

Proof. Since $d_{0}=-y_{0} \neq 0$, we can use $b, c, d$ as coordinates in a neighborhood of $\Phi\left(x_{0}, y_{0}, z_{0}\right)$. Clearly $\Phi$ has the inverse

$$
x=(b-1) / d, \quad y=-d, \quad z=(1-c) / d
$$

For $G$ supported in a fixed compact, we can, by exploiting a partition of unity, study $\Phi_{*}$ locally. Since $L_{\alpha, \mathrm{comp}}^{p}$ is left invariant by diffeomorphism (Theorem A.1.8), Proposition 4.1 implies that we need only study $\Phi_{*}$ for $G$ supported in a set of the form: $S_{M}=\left\{(x, y, z)|| x|\leqq M| y,\left|\leqq(2 M)^{-1},\right| z \mid \leqq M\right\}$. On this set, $b(x, y, z)=-x y+1$ lies in $\left[\frac{1}{2}, \frac{3}{2}\right]$, so we can use $a, b, d$ as coordinates. In fact, since the singular point $y=1$ corresponds to $b=1$, we use coordinates $u, v, w$ so that

$$
\Phi(x, y, z)=\left[\begin{array}{cc}
w & 1-v \\
\varphi(u, v, w) & u
\end{array}\right], \quad \varphi(u, v, w)=(w u-1) /(1-v) .
$$

Thus, we define the map $\Psi: \mathbb{R}^{3} \rightarrow \mathbb{R}^{3}$ by $\Psi(x, y, z)=(u, v, w)$ with $u=-y, v=x y$, $w=x y z-x-z$.

We use $r_{i}$ to denote $(x, y, z)$ and $\eta_{i}$ to denote $(u, v, w)$.

Proposition 4.2. (a) $O n \Psi\left[S_{M}\right],|v| \leqq M|u|$.

(b) the Jacobian $\left|\operatorname{det}\left(\partial \Psi / \partial r_{i}\right)\right|=|u(v-1)| \equiv J(u, v, w)$,

(c) the inverse map $\Psi^{-1}$ defined on $\Psi\left[S_{M}\right] /\{(u, v, w) \mid u=v=0\}$ obeys.

$$
\left\|\partial \Psi^{-1} / \partial \eta_{i}\right\| \leqq C\left(u^{2}+v^{2}\right)^{-1 / 2}
$$

(d) $\left|J(\eta)^{-1}\right| \leqq C\left(u^{2}+v^{2}\right)^{-1 / 2} ;\left|\nabla_{\eta}(1 / J)(\eta)\right| \leqq C\left(u^{2}+v^{2}\right)^{-1}$ on $\Psi\left[S_{M}\right]$.

Proof. (a) is immediate from the formula for $u, v$. It is easy to compute $\partial \Psi / \partial r_{i}$ and see that its determinant is $y(x y-1)=-u(v-1)$, proving (b). $\Psi^{-1}$ has the form $x=$ $-v / u, y=-u, z=-(1-v)^{-1}\left(w-v u^{-1}\right)$, and using the fact that $v / u$ is bounded on $\Psi\left[S_{M}\right]$, it is easy to prove that $\left\|\partial \Psi^{-1} / \partial \eta_{i}\right\| \leqq \widetilde{C}|u|^{-1}$. But since $|v| \leqq M|u|$, $|u|^{-1} \leqq\left(1+M^{2}\right)^{-1 / 2}\left(u^{2}+v^{2}\right)^{-1 / 2}$. Given this fact, (d) is a trivial computation using the form of (b). 
Since the singular points $\{r \mid y=0\}$ have Lebesgue measure zero, it is obvious that $\Phi_{*}(G)$ has a density, i.e. $\Phi_{*}(G)=\Phi^{\#}(G) d A$, where $\Phi^{\#}(G)$ is given by $\Phi^{\#}(G)(\eta)=$ $(1-v) T(G)(\eta)$ with $T(G)(\eta)=J(\eta)^{-1}\left(G \circ \Psi^{-1}\right)(\eta)$. The factor $(1-v)$, which comes from the fact that $d A=(1-v)^{-1} d u d v d w$, is $C^{\infty}$ and so unimportant. Thus, Theorem 3.7 is implied by:

Theorem 4.2. Let $\alpha>0$. Then, for all $p$ sufficiently close to $1, T$ maps $L_{\alpha}^{p}\left(S_{M}\right)$ to $L_{\beta}^{r}\left(R^{3}\right)$ for some $\beta>0, r>1$.

We will obtain this result as a corollary of Theorem 4.3 below. We note that $T$ does not map $C^{\infty}$ into $L^{p_{1}}$ since, if $G$ is $C^{\infty}$ and $G \equiv 1$ on $\widetilde{S}_{M}=\left\{r \| x \mid \leqq \frac{1}{2} M\right.$, $\left.|y| \leqq(4 M)^{-1},|z| \leqq \frac{1}{2} M\right\}$, then $T(G)(\eta)=J(\eta)^{-1}$ on $\Psi\left(\widetilde{S}_{M}\right)$ and $\int_{\Psi\left(\tilde{S}_{M}\right)}\left|\nabla\left[J(\eta)^{-1}\right]\right| d \eta=\infty$.

We introduce a family of maps, $0 \leqq \operatorname{Re} z<2$,

$$
T_{z}(G)(\eta)=J(\eta)^{-z}\left(G \circ \Psi^{-1}\right)(\eta)
$$

Theorem 4.3. Let $0 \leqq \alpha \leqq 1,1<p<\infty, 1<r<p$. Then $T_{z}$ is a bounded map from $L_{\alpha}^{p}\left(S_{M}\right)$ to $L_{\alpha}^{r}\left(R^{3}\right)$ so long as

$$
2 r^{-1}-p^{-1}>\max (1, \alpha+\operatorname{Re} z) .
$$

Proof. Suppose that $r, p$ are fixed with $2 r^{-1}-p^{-1}>1$. If we prove the result for $\alpha=0,1$ (with polynomial bounds in $\operatorname{Im} z$ on norms), then by the Stein interpolation theorem [16], the result holds for all $\alpha \in(0,1)$.

For $\alpha=0, \int|J(\eta)|^{-1}\left|F\left(\Phi^{-1} \circ \eta\right)\right|^{p} d u d v d w=\int|F(r)|^{p} d^{3} r$ so $F \in L^{p}$ implies that $J^{-1 / p} F \circ \Phi^{-1} \in L^{p}$. Thus, by Hölder's inequality, the $\alpha=0$ result holds if

$$
\int_{\Psi\left(\tilde{S}_{M}\right)}\left|J^{-z}\right|^{q}\left|J^{q / p}\right| d^{3} \eta<\infty
$$

when $\operatorname{Re} z<2 r^{-1}-p^{-1}$, where $q^{-1}=r^{-1}-p^{-1}$. To check this, we note that if $\operatorname{Re} z<2 r^{-1}-p^{-1}$, then

$$
q\left(\operatorname{Re} z-p^{-1}\right)<q\left(2 r^{-1}-2 p^{-1}\right) \leqq 2,
$$

so that (4.1) is equivalent to

$$
\int_{\Psi\left(\widetilde{S}_{M}\right)}|J|^{-a} d^{3} \eta<\infty \quad \text { if } \quad a<2
$$

Inequality (4.2) is easy to check if we note that $|J|^{-a} \leqq\left(u^{2}+v^{2}\right)^{-a / 2}$ on the region of integration.

For $\alpha=1$, we note that

$$
\nabla_{\eta}\left(T_{z}(G)\right)=J^{-z} \frac{\partial \Psi^{-1}}{\partial \eta}\left(\nabla_{r} G\right)\left(\Psi^{-1} \circ\right)+\nabla_{\eta}\left(J^{-z}\right)\left(G^{\circ} \Psi^{-1}\right) .
$$

Since $\left|\frac{\partial \Psi}{\operatorname{Lg}}\right| \leqq c J^{-1}$ and $\left|\nabla_{\eta}\left(J^{-z}\right)\right| \leqq c(|z|+1)\left|J^{-z-1}\right|$, we see that

$$
\left|\nabla_{\eta} T_{z}(G)\right| \leqq c|J|^{-\operatorname{Re} z-1}(|z|+1)\left[\left|G \circ \Psi^{-1}\right|+\mid\left(\nabla_{r}(G) \circ \Psi^{-1} \mid\right],\right.
$$

so that the exact same calculation as above proves the desired $\alpha=1$ result. 
Proof of Theorem 4.2. Without loss, we can shrink $\alpha$ so $\alpha<6$. By a Sobolev estimate, $L_{\alpha}^{p} \subset L_{\alpha / 2}^{p_{0}}$ so long as $1<p<p_{0} \equiv\left(1-\frac{1}{6} \alpha\right)^{-1}$. Let $r_{0}=(1-\alpha / 24)^{-1}$. Then $r_{0}<p_{0}$ and

$$
2 r_{0}^{-1}-p_{0}^{-1}=1+\frac{1}{12} \alpha>1+\frac{1}{13} \alpha .
$$

Since $L_{\alpha / 2}^{p_{0}} \subset L_{\alpha / 13}^{p_{0}}$, we conclude by Theorem 4.8 that $T_{z=1}$ map $L_{\alpha / 2}^{p_{0}}$ to $L_{\alpha / 13}^{r_{0}}$. We remark that by optimizing choices, we can take $\beta$ arbitrarily close to $\min (\alpha / 4,1)$.

\section{Appendix 1. Some Background on $L^{p}$ Sobolev Spaces}

In this appendix, we present, for the reader's convenience, some necessary background on the spaces $L_{\alpha}^{p}\left(R^{n}\right)$ discussed, e.g. in Stein [20], Calderon [4], and Chap. I of Taylor [22].

Definition. We say that $f \in L_{\alpha}^{p}(\alpha \geqq 0,1 \leqq p \leqq \infty)$ if and only if there exists $g \in L^{p}$ with $\hat{g}(k)=\left(1+k^{2}\right)^{\alpha / 2} \hat{f}(k)$. We set $\|f\|_{p ; \alpha} \equiv\|g\|_{p}$.

Equivalently, one can define the Bessel potential, $G_{\alpha}(x)$, by $\left(G_{\alpha} * g\right)^{\wedge}(k)=$ $\left(1+k^{2}\right)^{-\alpha / 2} \hat{g}(k)$, and then $L_{\alpha}^{p}=\left\{G_{\alpha} * g \mid g \in L^{p}\right\}$. As we will explain, there are special subtleties associated with the cases $p=1, \infty$. We can avoid these, since we will deal typically with $f$ of compact support, and for $\operatorname{such} f, L_{\alpha}^{p} \subset L_{\alpha}^{1}$ with $L_{\alpha}^{1} \subset L_{\alpha^{\prime}}^{p}$ for $p>1$ and $\alpha^{\prime}$ near $\alpha$. By this set of arguments, we typically lose a little bit on $\alpha$. There are surely places below where, by working harder, we could avoid this loss. Since for our purposes here the loss is irrelevant, we take the easy way out. One place that the special nature of $p=1, \infty$ occurs is

Theorem A.1.1. (see Calderon [4]). If $1<p_{0}, p_{1}<\infty, 0 \leqq \alpha_{0}, \alpha_{1}$, the complex interpolation spaces $\left(L_{\alpha_{0}}^{p_{0}}, L_{\alpha_{1}}^{p_{1}}\right)_{t}(0<t<1)$ are equivalent to $L_{\alpha_{z}}^{p_{z}}$ with $p_{z}^{-1}=t p_{1}^{-1}+$ $(1-t) p_{0}^{-1}, \alpha_{t}=t \alpha_{1}+(1-t) \alpha_{0}$. The same result holds if $L_{\alpha}^{p}\left(R^{v}\right)$ is replaced by $L_{\alpha}^{p}(\Omega)=\left\{f \in L_{\alpha}^{p}\left(R^{v}\right) \mid \operatorname{supp} f \subset \Omega\right\}$ for any open $\Omega$.

Since ([20], pg. 132), $G_{\alpha} \in L^{p}$ so long as $p<n /(n-\alpha)$ and $G_{\alpha} * G_{\beta}=G_{\alpha+\beta}$, Young's inequality implies a Sobolev estimate.

Theorem A.1.2. $L_{\alpha}^{p} \subset L_{\beta}^{q}$ if $q \geqq p, \beta \leqq \alpha$ and $p^{-1}-q^{-1}<(\alpha-\beta) n^{-1}$.

We will not need the more subtle result that one can have equality in the last requirement if $p>1, q<\infty$. It is not hard to see (e.g. [16], Sect. IX. 10) that the Fourier transform of $\left(1+k^{2}\right)^{\alpha / 2}$ is a distribution given by a smooth exponentially decaying function away from $x=0$. Thus, if $f \in L^{1}$ has compact support and $\hat{g}=$ $\left(1+k^{2}\right)^{\alpha / 2} \hat{f}$, then $g$ is a smooth exponentially decaying function away from a neighborhood of supp $f$. It follows that if $g \in L^{p}$, then $g \in L^{q}$ for all $q<p$. Thus:

Theorem A.1.3. If $f \in L_{\alpha}^{p}$ has compact support, then $f \in L_{\alpha}^{q}$ for all $q<p$ and $\|f\|_{q ; \alpha} \leqq C\|f\|_{p ; \alpha}$, where $C$ only depends on $q, \alpha$ and supp $f$.

The last two results imply that

Corollary A.1.4. $\bigcup_{1 \leqq p \leqq \infty, \alpha>0}\left\{f \in L_{\alpha}^{p} \mid \operatorname{supp} f\right.$ is compact $\}=\bigcup_{\alpha>0}\left\{f \in L_{\alpha}^{1} \mid \operatorname{supp} f\right.$ is compact\}.

It is for this reason that our basic result, Theorem 1.1, is stated in terms of $L_{\alpha}^{1}$. 
Example. The characteristic function of an interval has a Fourier transform $\hat{\chi}$ bounded by $(1+|k|)^{-1}$. Thus $\left(1+k^{2}\right)^{\alpha / 2} \hat{\chi} \in L^{2}$ so long as $\alpha<\frac{1}{2}$. It follows that $\chi \in L_{\alpha}^{2}$ for all $\alpha<\frac{1}{2}$ and so in $L_{\alpha}^{1}$ for all $\alpha<\frac{1}{2}$. Actually, a more careful analysis shows that $\chi \in L_{\alpha}^{1}$ for all $\alpha<1$.

The following result is a consequence of Theorem 3 on pg. 135 of [20]; see also pg. 31 of [22].

Theorem A.1.5. Let $1<p<\infty$. If $\alpha$ is an integer $k$, then $f \in L_{\alpha}^{p}$ if and only if for all multi-indices $|\beta| \leqq k, D^{\beta} f$ (distributional derivative) lies in $L^{p}$ and $\sum_{|\beta| \leqq k}\left\|D^{\beta} f\right\|_{p}$ is equivalent to $\|f\|_{p, \alpha}$.

The next few results are needed on $L_{\alpha}^{1}$. We begin with $L_{\alpha}^{p}, 1<p<\infty$, and then apply the strategy indicated above to get the $L_{\alpha}^{1}$ result.

Theorem A.1.6. If $g \in C^{\infty}$, with all derivatives bounded, then $f \mapsto g f$ maps $L_{s}^{p}$ to itself if $1<p<\infty$.

Proof. This follows from Theorem A.1.5 if $\alpha$ is an integer and for general $\alpha$ by interpolation.

Theorem A.1.7. Let $1<p<\infty$. If $f \in L_{\alpha}^{p}\left(R^{\mu}\right)$ and $g \in L_{\alpha}^{p}\left(R^{v}\right)$ and if $f \otimes g$ is the function $f(x) y(y)$ on $R^{\mu+v}$, then $f \otimes g \in L_{q}^{p}\left(R^{\mu+v}\right)$ and $\|f \otimes g\|_{p, \alpha} \leqq C(p, \alpha, \mu, v)\|f\|_{p, \alpha}\|g\|_{p, \alpha}$.

Proof. If $\alpha$ is an integer, this follows from Theorem. A.1.5 and for general $\alpha$ by bilinear interpolation.

Theorem A.1.8. Let $1<p<\infty$. Let $\Omega$ be a bounded open set in $R^{\mu}$, and let $\Phi$ be a diffeomorphism of $\Omega$ to a bounded open subset $\Omega^{\prime} \subset R^{\mu}$ which extends to a diffeomorphism of a neighborhood of $\bar{\Omega}$. Let $\Phi^{*}$ be defined from functions on $\Omega$ to functions on $\Omega^{\prime}$ by $\left(\Phi^{*} f\right)(x) f\left(\Phi^{-1}(x)\right)$. Then $\Phi^{*}$ is a bounded map of $L_{q}^{p}(R)$ to $L_{\alpha}^{p}\left(R^{\prime}\right)$.

Proof. By hypothesis, all derivatives of $\Phi$ and $\Phi^{-1}$ are bounded, so Theorem A.1.5 yields the result if $\alpha$ is an integer. We obtain general $\alpha$ by interpolation.

Let $L_{\alpha, \text { comp }}^{p}=\cup\left\{L_{\alpha}^{p}(\Omega) \mid\right.$ all bounded open $\left.\Omega\right\}$. We say $T$ from $L_{\alpha, \text { comp }}^{p}$ to $L_{\alpha, \text { comp }}^{q}$ is bounded if, for any $\Omega$, there is an $\Omega^{\prime}$ with $T\left[L_{\alpha}^{p}(\Omega)\right] \subset L_{\alpha}^{q}\left(\Omega^{\prime}\right)$ and $\|T f\|_{q, \alpha} \leqq$ $C(\Omega)\|f\|_{p, \alpha}$. We have the following analog of Theorem A.1.6:

Theorem A.1.9. If $g \in C^{\infty}$ with all derivatives bounded and $\alpha^{\prime}<\alpha$, then $f \mapsto g f$ maps $L_{\alpha, \text { comp }}^{1}$ to $L_{\alpha^{\prime}, \text { comp }}^{1}$.

Proof. By Theorem A.1.2, $L_{\alpha}^{1}$ lies in $L_{\alpha}^{p}$, for some $p>1$. Thus, by Theorem A.1.6, gf lies in $L_{\alpha^{\prime}}^{p}$. Finally, by Theorem A.1.2, $L_{\alpha^{\prime}, \text { comp }}^{p}$ is imbedded continuously in $L_{\alpha^{\prime}, \text { comp. }}^{1}$

The same reasoning implies our final results:

Theorem A.1.10. Let $\quad \alpha^{\prime}<\alpha$. Then $f \otimes g$ maps $L_{\alpha, \text { comp }}^{1}\left(R^{\mu}\right) \times L_{\alpha, \text { comp }}^{1}\left(R^{v}\right)$ to $L_{\alpha^{\prime}, \text { comp }}^{1}\left(R^{\mu+v}\right)$.

Theorem A.1.11. Under the hypothesis of Theorem A.1.8, $\Phi^{*}$ maps $L_{\alpha}^{1}(\Omega)$ to $\bigcap_{\alpha^{\prime}<\alpha} L_{\alpha^{\prime}}^{1}(\Omega)$. 


\section{Appendix 2. Some Background on $\operatorname{SL}(2, R)$ and its Sobolev Spaces}

In this appendix, we present for the reader's convenience some elementary facts about $\mathrm{SL}(2, R)$, including the proof of Theorem 3.6. $\mathrm{SL}(2, R)$ is, of course, all $2 \times 2$ real matrices $\left[\begin{array}{ll}a & b \\ c & d\end{array}\right]$ of determinant 1 , i.e. $a d-b c=1$. It is, of course, a three dimensional manifold covered by two "coordinate patches," $P_{1}=\{(a, b, c) \mid a \neq 0\}$ and $P_{2}=\{(a, b, d) \mid b \neq 0\}$. By the standard Jacobian formula, $\operatorname{SL}(2, R)$ acting on all $2 \times 2$ matrices $\left[\begin{array}{ll}x & y \\ w & z\end{array}\right]$ by either left or right multiplication leaves the measure $d x d y d w d z$ invariant, so the measure $\delta(a b-c d) d a d b d c d d$ is a left and right invariant Haar measure on SL $(2, R)$, i.e. Haar measure has the form $|a|^{-1} d a d b d c$ on $P_{1}$ and $|b|^{-1}$ dadbdd on $P_{2}$.

We define maps $\tau_{A}^{L}, \tau_{A}^{R}$ for $A \in \operatorname{SL}(2, R)$ on functions, $f$, on $\operatorname{SL}(2, R)$ by $\left(\tau_{A}^{L} f\right)(B)=f\left(A^{-1} B\right)$ and $\left(\tau_{A}^{R} f\right)(B)=f(B A)$. A vector field $X$ is called left (respectively right) invariant if it commutes with all $\tau_{A}^{L}$ (respectively all $\left.\tau_{A}^{R}\right)$. It is standard fact that the map $X \mapsto X_{1}$ sets up a bijection between the left invariant (or right invariant) vector fields and the tangent space, $\operatorname{SL}(2, R)$ at the identity, 1.

Pick an arbitrary basis $X_{1}, X_{2}, X_{3}$ for $\operatorname{SL}(2, R)$, and let $X_{i}^{L}$ (respectively $X_{i}^{R}$ ) be the left invariant (respectively right invariant) vector fields equal to $X_{i}$ at the identity. Let $\Delta^{L}=\sum_{i=1}^{3}\left(X_{i}^{L}\right)^{2}$ and similarly for $\Delta^{R}$. If $g_{0}$ is the metric on $\operatorname{SL}(2, R)$ in which $X_{i}$ is an orthonormal basis and $g^{L}$ (respectively $g^{R}$ ) is the unique left (respectively right) invariant metric on $\operatorname{SL}(2, R)$ agreeing at 1 with $g_{0}$, then $\Delta^{L}$ (respectively $\Delta^{R}$ ) is the Laplace-Beltrami operator associated to $g^{L}$ (respectively $g^{R}$ ). Because of the invariance of the metric, it is easy to see that there is a $\delta$ independent of $A$ so that every speed 1 geodesic starting at $A$ can be run for time $\delta$, and from this one obtains the completeness of the metrices. Standard theorems on the self adjointness of Laplace-Beltrami operators $[5,21]$ thus imply:

Theorem A.2.1. $\Delta^{L}$ and $\Delta^{R}$ are essentially selfadjoint on $C_{0}^{\infty}(\operatorname{SL}(2, R))$.

We define the Sobolev space $H^{s}$ for $s>0$ to be the set of $u \in L^{2}$ which lie in $D\left(\left(\Delta^{L}\right)^{s / 2}\right)$ with the norm $\left\|\left(1-\Delta^{L}\right)^{s / 2} u\right\|=\|u\|_{s}$. For $s<0$, the space is just the completion of $L^{2}$ in this same norm. By Calderon's theory [4], the complex interpolation spaces $\left(H^{s_{0}}, H^{s_{1}}\right)_{\theta}$ are just $H^{\theta s_{1}+(1-\theta) s_{0}}$. Any distribution of compact support lies in some $H^{s}(-\infty<s<\infty)$. We note that by a standard partition of unity argument, for any compact $K$, and any bounded open neighborhood $U$ of $K$, and any $s>0,\|T\|_{H^{-s}} \leqq C, \sup \left\{T(\varphi) ; \operatorname{supp} \varphi \subset U,\|\varphi\|_{H^{s}}=1\right\}$ for all $T$ supported in $K$. Thus, we can associate the dual of $H_{\text {comp }}^{s}$ with $H_{\text {loc }}^{-s}$ in the sense that a bounded map from $H_{\text {comp }}^{s}$ to $H_{\text {comp }}^{t}$ has a dual mapping $H_{\text {loc }}^{-t}$ to $H_{\text {loc }}^{-s}$. If such a map is given by convolution by a compactly supported distribution, then the dual map also takes $H_{\text {comp }}^{-t}$ to $H_{\text {comp }}^{-s}$.

The standard theory of Sobolev spaces on manifolds implies that for each bounded open set, $K$, and each even integer, $n$, the norms $\|\cdot\|_{n}, \sum_{|\alpha| \leqq n}\left\|D^{\alpha} u\right\|_{2}$ and $\left\|\left(1-\Delta^{R}\right)^{n / 2} u\right\|_{2}$ are equivalent norms on $\left\{u \mid u \in C_{0}^{\infty}(K)\right\}$ (this result remains true for 
all integers $n$ and for non-integral $n$ if a suitable replacements for $\left\|D^{\alpha} u\right\|_{2}$ is found; we won't need that).

For $p>1$, we define $L_{\alpha}^{p}$ to be the completion of $C_{0}^{\infty}$ in the norm $\left\|\left(1-\Delta^{L}\right)^{\alpha / 2} f\right\|_{p}$. It is proven by Strichartz [21] that $\left(L_{\alpha_{0}}^{p_{0}}, L_{\alpha_{1}}^{p_{1}}\right)_{\theta}=L_{\alpha_{\theta}}^{p_{\theta}}$, where $\alpha_{\theta}=\theta \alpha_{1}+(1-\theta) \alpha_{0}$, $p_{\theta}^{-1}=\theta p_{1}^{-1}+(1-\theta) p_{0}^{-1}$. It is again standard theory of Sobolev spaces on manifolds that if $u$ has compact support, that $u \in L_{\alpha}^{p}$ if and only if it in the standard $L^{p} \alpha\left(R^{v}\right)$ in a local coordinate system about each point.

Convolution is defined by $(f * g)(A)=\int f\left(A B^{-1}\right) g(B) d B$. If $f, g \in L^{1}$, then by Fubini's theorem the integral converges for a.e. $A$ and we define convolution initially on $L^{1} \times L^{1}$. Since the Haar measure is both left and right invariant, $d B$ is invariant under $B \rightarrow B^{-1}$ and thus, by a change of variables

$$
(f * g)(A)=\int f(C) g\left(C^{-1} A\right) d C .
$$

These equations can be written in the form

$$
\begin{aligned}
f * g & =\int g(B)\left(\tau_{B^{-1}}^{R^{\prime}} f\right) d B \\
& =\int f(C)\left(\tau_{C}^{L} g\right) d C
\end{aligned}
$$

Since $\Delta^{\#}$ commutes with $\tau^{\#}$ (for \#=R or $L$ ), we see that for $f, g \in C_{0}^{\infty}$,

$$
\left(1-\Delta^{R}\right)^{\alpha / 2}(f * g)=\left[\left(1-\Delta^{R}\right)^{\alpha / 2} f\right] * g,
$$

and

$$
\left(1-\Delta^{L}\right)^{\alpha / 2}(f * g)=f *\left[\left(1-\Delta^{L}\right)^{\alpha / 2} g\right] .
$$

It is easy to see that if $f, g \in L^{1}$, then the measure convolution (defined in the text) of $f d A$ and $g d A$ is $(f * g) d A$. If $f \forall(A)=f\left(A^{-1}\right)$ and $\langle f, g\rangle^{\sim}=\int f(A) g(A) d A$ and $f, g, h \in C_{0}^{\infty}$, then

$$
\langle f, h * g\rangle^{\sim}=\langle h \nvdash * f, g\rangle^{\sim}=\langle f * g \natural, h\rangle^{\sim}
$$

by a change of variables. This allows one to define the convolution of a distribution of a compact support and a $C^{\infty}$ function of compact support (by $(\psi * T)(\varphi)=$ $T(\psi \bullet * \varphi)$ ), and to check that this convolution is again a $C^{\infty}$ function of compact support. Thus, one can define the convolution of any two distributions of compact support, and so $f * g$ for any $f, g \in \bigcup_{s=-\infty}^{\infty} H_{\text {comp }}^{s}$.

Theorem A.2.2. (= Theorem 3.6(a)). Let $T \in L_{s}^{p}, 1<p ; 0 \leqq s \leqq 1$ have compact support, and $f \in H_{\text {comp. Then }}^{t}$

$$
\|T * f\|_{H^{s+t}} \leqq C\|T\|_{L_{s}^{p}}\|f\|_{H^{t}},
$$

where $C$ depends only on $s, t$ and the supports of $f$ and $T$.

Proof. We claim first that we only prove here the result for $t \geqq 0$. For the duality (for $H_{\text {comp }}^{t}$ as discussed above) and (A.3) imply the result for $t \leqq-s$, and we can interpolate to obtain the result for all $t$. Also, by interpolation we need only prove the result for $s$ fixed, $t$ a non-negative even integer, and then for such $t$ and $s=0$ or 2 . 
By (A.1b) and the fact that $\tau^{L}$ is an isometry on each $H^{t}$, if $f \in L^{1}$ and $g \in H^{t}$ :

$$
\|f * g\|_{H^{t}} \leqq\|f\|_{1}\|g\|_{H^{t}},
$$

and so if $f \in L^{p}$ has support in a compact set $K$ (so $f \in L^{1}$ ),

$$
\|f * g\|_{H^{t}} \leqq C\|f\|_{p}\|g\|_{H^{t}}
$$

where $C$ depends only on $K$. Thus, if $s=0,2, t=0,2,4, \ldots$ and $T \in L_{s}^{p}$, then

$$
\left\|\left(1-\Delta^{R}\right)^{s / 2}(T * g)\right\|_{H^{t}}=\left\|\left[\left(1-\Delta^{R}\right)^{s / 2} T * g\right]\right\|_{H^{t}} \leqq C\left\|\left(1-\Delta^{R}\right)^{s / 2} T\right\|_{p}\|g\|_{H^{t}} .
$$

But as we have already remarked, the usual theory of Sobolev spaces on manifolds implies that on a fixed compact $\left\|\left(1-\Delta^{R}\right)^{s / 2} T\right\|_{p}$ and $L_{s}^{p}$ are equivalent norms, and that $\left\|\left(1-\Delta^{R}\right)^{s / 2} h\right\|_{H^{t}}$ and $H^{t+s}$ are equivalent norms.

Theorem A.2.3. ( $\equiv$ Theorem 3.6(b)). $H_{\text {comp }}^{s} * H_{\text {comp }}^{t} \subset H_{\text {comp }}^{s+1}$.

Proof. The previous argument extended to general positive $s$ yields the result for all $t$ and $s \geqq 0$. By duality (using (A.3)) for any $t, * H_{\text {comp }}^{t}$ maps $H_{\text {comp }}^{-s-t}$ to $H_{\text {comp }}^{-s}$ for all $s>0$, i.e. we have the original result if $s+t \leqq 0$. That is, we know the result for all pairs $\{\langle s, t\rangle \mid s \geqq 0$ or $s+t \leqq 0\}$. Thus, for $t$ fixed we directly have all $s$ if $t \leqq 0$ and we have all $s$ with $s$ in $(-\infty,-t]$ or $[0, \infty)$ if $t>0$. By interpolating in $s$, we obtain the result for all $s$.

\section{Appendix 3. A Theorem of Halperin}

In this appendix, we will prove

Theorem A.3.1. Let $d \eta(v)=\theta \delta(v-a)+(1-\theta) \delta(v-b) ; 0<\theta \leqq \frac{1}{2}$. Then $k(E)$ is not Hölder continuous of any order $\alpha$ larger than

$$
\alpha_{0}=2|\log (1-\theta)| / \operatorname{Arccosh}\left(1+\frac{1}{2}|a-b|\right) .
$$

Remarks. 1. This result is essentially due to Halperin [23], whose strategy we follow quite closely. For reasons given in the introduction, we include it here.

2. Notice that $\alpha_{0} \downarrow 0$ as either $\theta \rightarrow 0$ or $|a-b| \rightarrow \infty$. Thus one cannot hope to prove Hölder continuity of any preassigned order.

3. Once $|a-b|>2.25$, we see that $\alpha_{0}<1$ for all $\theta$.

4. As we will explain, there is reason to believe that $d k$ has a singular continuous component for suitable $a, b, \theta$.

We will need a version of Temple's inequality:

Lemma A.3.2. Let $A$ be a selfadjoint operator. Suppose that $\left\{f_{i}\right\}_{i=1}^{k}$ is an orthonormal set obeying

(i) $\left\|\left(A-E_{0}\right) f_{i}\right\| \leqq \varepsilon$,

(ii) $\left(f_{i}, A f_{j}\right)=\left(A f_{i}, A f_{j}\right)=0$ all $i \neq j$,

for some $E_{0}$, $\varepsilon$. Then the spectral projection $P_{\left[E_{0}-\varepsilon, E_{0}+\varepsilon\right]}(A)$ has range at least $k$.

Proof. Let $V$ be the span of the $f_{i}$. An elementary calculation shows that for any $f \in V$, $\left\|\left(A-E_{0}\right) f\right\| \leqq \varepsilon\|f\|$. If $\operatorname{dim} \operatorname{Ran} P_{\left[E_{0}-\varepsilon, E_{0}+\varepsilon\right]} \leqq k-1$, we can find $f \in V$ orthonormal to it, in which case $\left\|\left(A-E_{0}\right) f\right\|>\varepsilon\|f\|$. This contradiction establishes the result. 
Proof of Theorem A.3.1. As a warm-up result, we will prove the theorem with $\alpha_{0}$ replaced by the larger number,

$$
\alpha_{1}=2|\log (1-\theta)| / \operatorname{Arc} \sinh \left(\frac{1}{2}|a-b|\right) .
$$

Without loss of generality, we can suppose that $a>b$, since $H_{0}+V$ and $-H_{0}+V$ are unitarily equivalent. Consider an infinite volume potential, $\widetilde{V}$, with $\tilde{V}(0)=a$, $\widetilde{V}(n)=b(n \neq 0)$. It is not hard to see that $H_{0}+\widetilde{V}$ has an eigenfunction

with

$$
\varphi(n)=e^{-k|n|}
$$

$$
k=\operatorname{Arcsinh}\left(\frac{1}{2}|a-b|\right)
$$

and eigenvalue $E_{0}=a+2 e^{-k}$. For each $L$, we can find a normalized $\varphi_{L}$ supported on $\{n|| n \mid \leqq L-1\}$ so that

$$
\left\|\left(H-E_{0}\right) \varphi_{L}\right\| \leqq 2 e^{-k(L-1)},
$$

for we need only take $\varphi_{L}(n)=N_{L}^{-1} \varphi(n)$ for $|n| \leqq L-1 . N_{L}>1$ and $\left(H-E_{0}\right) \varphi_{L}(j)=0$ if $j \neq \pm(L-1) ;=-e^{-k L}$ if $j= \pm(L-1)$ and $=e^{-k(L-1)}$ if $j= \pm L$.

Now fix a "typical" potential $V_{\omega}$, "typical" in the sense that $\lim _{L \rightarrow \infty} L^{-1}$ (\# of $e v$ of $\left.H_{\omega}^{L} \leqq E\right) \rightarrow k(E)$ for each $E$, and in a sense made precise below. Fix $L_{0}$ and take $L=m\left(2 L_{0}+1\right)$ with $m=1,2, \ldots$ Break $[0, L-1]$ into $m$ blocks $\left[0,2 L_{0}\right],\left[2 L_{0}+1\right.$, $\left.4 L_{0}+1\right], \ldots$ Suppose that $n_{m}$ of these blocks have $V_{\omega}$ equal to $a$ in the center and $b$ at the other $2 L_{0}$ sites. Let $f_{1}, \ldots, f_{n_{m}}$ be the function $\varphi_{L_{0}}$ translated to be centred at the center of the special blocks. Then

$$
\left\|\left(H_{\omega}^{L}-E_{0}\right) f_{j}\right\| \leqq 2 e^{-k\left(L_{0}-1\right)}
$$

and since $H f_{j}$ is supported in the $j^{\text {th }}$ special block, the other hypotheses of the Temple inequality hold. We conclude that $H_{\omega}^{L}$ has at least $n_{m}$ eigenvalues in the interval $\left.\left[E_{0}-2 e^{-k\left(L_{0}-1\right)}, E_{0}+2 e^{-k\left(L_{0}-1\right)}\right)\right]$. Thus

$$
k\left(E_{0}+2 e^{-k\left(L_{0}-1\right)}\right)-k\left(E_{0}-2 e^{-k\left(L_{0}-1\right)}\right) \geqq \lim _{m \rightarrow \infty} L^{-1} n_{m}=\left(2 L_{0}+1\right)^{-1} \lim _{m \rightarrow \infty} m^{-1} n_{m}
$$

The law of large numbers tells us that for a typical $\omega, m^{-1} n_{m} \rightarrow \operatorname{Prob}(V(0)=a$, $\left.V( \pm 1)=\cdots=V\left( \pm L_{0}\right)=b\right)=\theta(1-\theta)^{2 L_{0}}$. Thus, letting $\delta_{L_{0}}=2 e^{-k\left(L_{0}-1\right)}$, we see that

$$
k\left(E_{0}+\delta_{L_{0}}\right)-k\left(E_{0}-\delta_{L_{0}}\right) \geqq\left(2 L_{0}+1\right)^{-1} \theta(1-\theta)^{2}\left(\frac{1}{2} \delta_{L_{0}}\right)^{\alpha_{1}} .
$$

Since $L_{0}^{-1}=0\left(\left[\log \delta_{L_{0}}^{-1}\right]^{-1}\right)$ and $\delta_{L_{0}} \downarrow 0$, we see that $k$ cannot be Hölder continuous of any order $\alpha>\alpha_{1}$.

Clearly the same argument works for any eigenvalue of an operator with potential $V^{\#}(n) \equiv b$ for $|n|$ large. If $k^{\#}$ is the rate of decay of the eigenfunction as $|n| \rightarrow \infty$, then one cannot be Hölder continuous of order larger than $\alpha^{\#}=$ $2|\log (1-\theta)| / k^{\#}$. If $E^{\#}>b+2$ is the eigenvalue and $V^{\#}=b$ for $n$ large, then $k^{\#}$ obeys $2 \cosh k^{\#}=\left|E^{\#}-b\right|$. If we look at the largest eigenvalue $e_{j}$ of the potential $V(n)=a$ (respectively $b$ ) if $|j|<n$ (respectively $\geqq n$ ), then $e_{j} \rightarrow a+2$ as $j \rightarrow \infty$. Thus

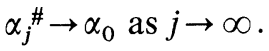

Remarks. 1. This argument is restricted to one dimension, for in higher dimensions the value of $\left\|\left(H_{L}-E_{0}\right) \varphi_{L}\right\|$ still goes as $e^{-k L_{0}}$ but the probability of a given 
configuration with one $V(n)=a$ goes as $\theta(1-\theta)^{\left(2 L_{0}+1\right)^{d}-1} \sim e^{-c L_{0}^{d}}$. One can speculate that just as the normal singularities of the periodic potential density of states get less severe as dimension increases, so might it happen that the minimal smoothness Anderson model density of states gets better as dimension increases.

2. The argument is valid for continuum models $-\left(d^{2} / d x^{2}\right)+V(x)$. For example, if $V_{0}$ and $V_{1}$ have period 1, and if in each cell $[n, n+1], V$ is either $V_{0}$ or $V_{1}$ with probability $\theta$ and $(1-\theta)$, and if $\sigma\left(-\left(d^{2} / d x^{2}\right)+V_{0}\right) \neq \sigma\left(-\left(d^{2} / d x^{2}\right)+V_{1}\right), k$ will not be $C^{1}$ if $\theta$ is suitably chosen.

Let us give a heuristic argument suggesting that $d k$ has a singular component. We suppose that the system only has localized states and that in a certain small energy region they decay as $e^{-\gamma|n|}$ in this energy region in a large box of size $\left(2 L_{0}+1\right)$, roughly half of them should be localized in the middle half of the box, and so should be of size at most $e^{-\gamma L_{0} / 2}$ at the edges. Thus, using the construction above, we find half the eigenvalues in this region in intervals of size $C e^{-\gamma L_{0} / 2}$. But the number of intervals is no more than the number of eigenvalues of all possible potentials in a box of side $2 L_{0}+1$, is $\left(2 L_{0}+1\right) 2^{2 L_{0}+1}$. Thus we have concentrated half the eigenvalues in a union of intervals of Lebesgue measure $\left(2 L_{0}+1\right) 2^{2 L_{0}+1} e^{-\gamma L_{0} / 2} \rightarrow 0$ as $L_{0} \rightarrow \infty$ if $e^{-\gamma}<1 / 16$.

Nieuwenhuizen and Luck [25] have recently made a nonrigorous but very illuminating study of the Anderson model with two valued potential.

Acknowledgement. It is a pleasure to thank Tom Spencer for stimulating our interest in this question, and Eli Stein for the useful remark that it is often easier to control convolutions on $\operatorname{SL}(2, R)$ with one's "bare hands" rather than by using the non-commutative Fourier transform.

\section{References}

1. Atkinson, F.: Discrete and continuous boundary problems. New York: Academic Press 1964

2. Avron, J., Simon, B.: Almost periodic Schrödinger operators. II. The integrated density of states. Duke Math. J. 50, 369-391 (1983)

3. Benderskii, M., Pastur, L.: On the spectrum of the one dimensional Schrödinger equation with a random potential. Mat. Sb. 82, 245-256 (1970)

4. Calderon, A.: Intermediate spaces and interpolation. Studia Math. Spec. Series 1, 31-190 (1983)

5. Chernoff, P.: Essential self-adjointness of powers of generators of hyperbolic equations. J. Funct Anal 12, 401-414 (1973)

6. Craig, W., Simon, B.: Subharmonicity of the Lyaponov index. Duke Math. J. 50, 551-560 (1983)

7. Fukushima, M.: On the spectral distribution of a disordered system and the range of a random walk. Osaka J. Math. 11, 73-85 (1974)

8. Furstenberg, H.: Noncommuting random products. Trans. Am. Math. Soc. 108, 377-428 (1963)

9. Furstenberg, H.: Boundary theory and stochastic processes on homogeneous spaces, pp. 193-229, Proc. AMS Summer Institute on Homogeneous Spaces. Providence, RI: AMS 1973

10. Herbert, D., Jones, R.: Localized states in disordered systems. J. Phys. C4, 1145 (1971)

11. Kirsch, W., Martinelli, F.: On the density of states of Schrödinger operators with a random potential. J. Phys. A15, 2139-2156 (1982)

12. LePage, E.: Empirical distribution of the eigenvalues of a Jacobi matrix, pp. 309-367. In Probability measures on groups, VII, Springer Lecture Notes Series 1064. Berlin, Heidelberg, New York: Springer 1983

13. Mezincescu, G.: Bounds on the integrated density of electronic states for disordered Hamiltonians, IPTM (Bucharest) preprint 
14. Nagai, H.: On an exponential character of the spectral distribution function of a random difference operator. Osaka J. Math. 14, 111-116 (1977)

15. Pastur, L.: Spectral properties of disordered systems in one-body approximation. Commun. Math. Phys. 75, 179 (1980)

16. Reed, M., Simon, B.: Methods of modern mathematical physics, II. Fourier analysis, selfadjointness. New York: Academic Press 1975

17. Romerio, M., Wreszinski, W.: On the Lifshitz singularity and the tailing in the density of states for random lattice systems. J. Stat. Phys. 21, 169 (1979)

18. Schmidt, H.: Disordered one-dimensional crystals. Phys. Rev. 105, 425-441 (1957)

19. Simon, B.: Lifshitz tails for the Anderson model, Lifshitz Memorial Issue of J. Stat. Phys. 38, 65-76 (1985)

20. Stein, E.: Singular integrals and differentiability properties of functions. Princeton, NJ: Princeton University Press 1970

21. Strichartz, R.: Analysis of the Laplacian on a complete Riemannian manifold. J. Funct. Anal. 52, 48-79 (1983)

22. Taylor, M.: Pseudodifferential operators. Princeton, NJ: Princeton University Press 1981

23. Thouless, D.: A relation between the density of states and range of localization for one dimensional random systems. J. Phys. C5, 77-81 (1972)

24. Halperin, B.: Properties of a particle in a one-dimensional random potential. Adv. Chem. Phys. 31, 123-177 (1967)

25. Nieuwenhuizen, T. Luck, J.: Singular behavior of the density of states and the Lyaponov coefficient in binary random harmonic chains. J. Stat. Phys. (to appear)

Communicated by T. Spencer

Received February 22, 1985; in revised form April 5, 1985 
